\title{
Harnessing Artificial Intelligence and Machine Learning in Biomedical Applications with the Appropriate Regulation of Data
}

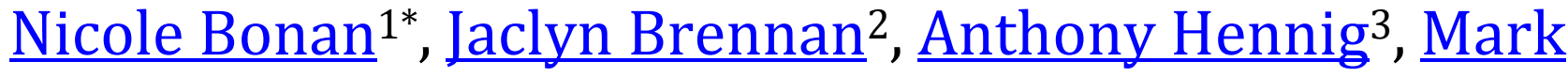 Alexander Kaltenborn 4,5}

${ }^{1}$ The George Washington University Institute for Biomedical Sciences, Washington, DC

2The George Washington University Department of Biomedical Engineering, Washington, DC

3The George Washington University Department of Engineering Management and Systems Engineering, Washington, DC

${ }^{4}$ The George Washington University Department of Physics, Washington, DC

${ }^{5}$ Los Alamos National Laboratory Computational Physics and Methods Group, Los Alamos, New Mexico https://doi.org/10.38126/ISPG180102

*Corresponding author: nbonan@gwmail.gwu.edu

Keywords: artificial intelligence; machine learning; software as a medical device; FDA; FDCA; database

Executive Summary: Medical devices and systems are increasingly relying on software using artificial intelligence (AI) and machine learning (ML) algorithms to increase efficiency, provide better diagnoses, and increase the quality of care for patients. AI- and ML-based devices and systems have an advantage over traditional medical device systems because they are designed to learn and improve using large databases of actual or simulated patient data. However, the use of these datasets could introduce harmful biases to certain populations, restrict economic development if policy were to change in the future, and negatively impact healthcare. We recommend amending the Food Drug and Cosmetic Act to explicitly direct the Secretary of Health and Human Services to regulate databases used by AI systems and require that the premarket review of medical databases includes assessments of potential bias and security.

\section{The use of data in artificial intelligence}

AI is defined as "any artificial system that performs tasks under varying and unpredictable circumstances without significant human oversight, or that can learn from experience and improve performance when exposed to data sets" (NDAA 2018). In the healthcare industry, AI systems have the potential to reduce the development time and cost of life-saving medical innovations. ML is an application of AI that provides systems the ability to automatically learn and improve from experience without being explicitly programmed. ML systems are initially trained by model datasets, and the quality of the training data directly dictates the quality and performance of the ML system. The ability of these systems to further adapt and improve for biomedical applications relies on accurate, representative, and continuous data from patient databases. The biomedical application that we focus on in this memo is "software as a medical device" (SaMD), which is one of three types of medical device-related software. SaMD, as defined by the International Medical Device Regulators Forum (IMDRF), is "software intended to be used for one or more medical purposes that perform these purposes without being part of a hardware medical device" (IMDRF, 2013). SaMD can perform a wide variety of tasks, such as providing information to diagnose or treat a disease (e.g., automated lung segmentation of high-resolution computed tomography) to replacing, modifying, or supporting an anatomical process (e.g., a pacemaker). 


\section{Database management in artificial intelligence}

\section{i. Importance of database management}

In order for AI and ML to effectively increase efficiency, provide better diagnoses, and increase patient quality of care, it is important to use optimally designed databases. For biomedical applications, ethical curation of these databases requires that designers both understand the key data involved in good biomedical decision-making and ethically manage patient data (Shrestha 2019). In 2019, the FDA issued a discussion paper detailing how SaMD could be approved as a medical device, and the Agency stated its intention of assessing "the culture of quality and organizational excellence of a particular company with reasonable assurance of the high quality of their software development, testing, and performance monitoring of their products" (USFDA 2019). This statement indicates the FDA's awareness of potential issues related to database design, and the proposed guidelines suggest a framework for safety and efficacy. However, they do not implement an appropriate regulatory framework for the specific data and databases that are utilized by the software. By omitting database regulation and thus data input, the FDA still risks approving devices that could reinforce bias, cause patient harm, and undermine patient and provider autonomy (Price 2017).

\section{ii. Examples of mismanaged databases}

Without database oversight, problems such as data "silo-ing" (i.e., collected information is sequestered or incompatible with other data systems), biased data analyzing (i.e., racial profiling, gender discrimination, improper representations of reality), or even data privacy breaching (e.g., GlaxoSmithKline's 2018 access to 23andMe genetic data or Equifax's 2017 data breach) can exist in AI/ML-based systems (Araujo 2020). The risks associated with poor database management are ever heightened in today's global pandemic, as the world struggles with control over COVID-19. As scientists and engineers come up with new ways to detect and, therefore, prevent the spread of COVID-19 cases, a number of data-driven algorithms are being developed. For example, researchers at MIT have found AI can detect asymptomatic COVID-19 infections through cell phone-recorded coughs, and various other AI models are sifting through patient data on symptoms by analyzing chest X-rays and CT scans (Laguarta 2020,
Borkowski 2020). While these algorithms are benevolently designed to help pull us out of this global pandemic, the lack of protections on these datasets is a major cause for concern.

\section{Regulation and legislation}

\section{i. Current legislation}

Currently, there is no comprehensive federal law that governs data privacy in the United States. To date, there have been 183 bills introduced since 1979 that mention AI (Congress.gov). Of those, only two related to healthcare, two to transportation, and five to national defense and security have become law (Library of Congress). The introduction of the Algorithmic Accountability Act (S.1108/H.R.2231) in 2019 reflects timely considerations for consumer rights and protections in our current high-tech era. This legislation would apply to companies that use AI and ML-based systems to make predictions or conclusions about their consumer base. It would require the companies to provide an assessment detailing measures that were taken to minimize bias, discrimination, and security and data privacy concerns in their software. This bill would directly apply to companies that create SaMD. The Algorithmic Accountability Act is still in its infancy, as it has not yet passed the House or Senate.

Still, the traditional paradigm of medical device (and SaMD) regulation by the FDA does not require reapproval of a medical device after its initial approval (FDA, 2020). This is fundamentally at odds with the nature of ML-based algorithms, which change and improve over time. This also fails to encompass the evolving databases necessary for training effective and unbiased AI/ML decision-making, especially in healthcare (Andel 2012, Ernst 2018, Congress 2019). Therefore, a solution to SaMD regulation would require a system for managing and regulating a this constantly evolving software and its input data.

\section{ii. Opportunities}

To combat the problems associated with poor database oversight in the context of AI, there exist several possible legislative options. First, the National Academies of Sciences, Engineering, and Medicine (NASEM) could give external scientific expertise and provide guidelines for AI to the FDA. NASEM has a long history of partnering with the federal government to provide scientific guidance for 
determining policies and regulation standards (Blair 2016, NASEM 2020). Second, Congress could pass an enabling act authorizing the creation of a new legislative agency for the oversight and regulation of AI systems and databases. Congress has previously recognized the need to establish new agencies to address paradigm shifts in society (e.g., establishment of NASA and the EPA). Third, the Federal Food, Drug, and Cosmetic Act (FDCA) could be amended to explicitly designate a sub-department of the FDA to regulate databases that are used by AI systems by requiring assessments of potential biases, similar to the proposed Algorithmic Accountability Act. This option would not only provide robust regulation of the device itself but also address the lack of re-approval in FDA's current medical device approval process by requiring reoccurring assessments.

\section{Recommendation}

We recommend that Congress take immediate action to amend the FDCA to include databases that support AI- and ML- based medical software such that the FDA would have the ability to inspect and regulate the use of this technology. This would include a) explicitly directing the Secretary of Health and Human Services to designate a component of the FDA to regulate databases that are utilized by AI systems in the manufacturing, distributing, and administering of a drug, device, diagnosis, or biological products and b) requiring that the premarket review includes assessments of potential bias, data silo-ing, privacy breaches, and the confirmation that the database is for medical purposes only.

\section{i. Stakeholders for the given recommendation}

This policy recommendation involves numerous stakeholders that will need to work together to make this action successful. First and foremost, we are asking for the FDA to take on a new set of responsibilities, which will require public health experts, data scientists, and engineers working together to develop ways of ensuring that AI and ML algorithms are accountable and their databases minimize the unintended effects of collecting and utilizing massive datasets. Second, this course of action will require private industry in both the biomedical and big data fields to participate in the regulation process and provide feedback on these processes, especially on the best practices for developing SaMD and checking SaMD and databases for and mitigating bias, data silo-ing, and privacy breaches. There are several large corporations already working on developing AI and ML platforms for public health, such as Microsoft's Biomedical ML division and IBM's Watson Machine Learning Group. During the COVID-19 pandemic, this number has only grown, with large technology companies such as Fitbit, Inc. running their own biomedical studies on the impact of the novel coronavirus on their user's health (Heneghan 2020). The companies in this space will benefit from reducing uncertainty in the regulatory environment that can negatively affect operations and products, and the consumers of these companies' products can be reassured that they can continue to benefit from the regulated services that they have come to expect. Finally, and of greatest importance, this action will protect the private biomedical data of individuals while protecting individuals from unintended consequences of big data, both in and out of hospitals (Timan, 2019).

\section{ii. Policy trade-offs}

There exist a few policy trade-offs for this option. In order to make this amendment work, specific expertise is required to understand the complexities of AI systems and their relationships with databases. Similar to the Federal Aviation Administration dealing with the growing complexity of aeronautics, the FDA may end up partially relying on industry experts to self-regulate or participate in the regulation process (Downer 2010). This might actually be a benefit moving into the future as the nature of these devices and the necessary regulations change. Thus, the next steps toward amending the FDCA should include the creation of a committee composed of legislators as well as experts in AI, ML, and biomedical science.

\section{Conclusion}

By inspecting and regulating these databases, the FDA will take the first step toward ensuring that AI and ML biomedical software systems are well trained and their outputs are efficient, provide better diagnoses, and increase patient quality of care. Health care costs from preventable medical errors account for 200,000 lost lives and nearly $\$ 20$ billion each year but having inspected and well-regulated medical software can reduce this impact (Andel 2012). Secondly, instead of deferring this option into the future, taking this option now provides more predictability and regularity to business and 
healthcare providers already developing this technology, potentially saving the nation wasted research and development costs. Finally, this option is similar to options taken by other agencies with their own applications of AI and ML software, but it imparts timely nationwide federal regulation to ensure uniform adherence to the protection of all Americans' unalienable rights (Ernst 2018).

\section{Appendix}

After this article was written, the FDA released an AI/ML-based Action Plan, published January 12, 2021. Included are two sections relating to issues presented in this article: Good Machine Learning Practice (GMLP) and Regulatory Science Methods Related to Algorithm Bias and Robustness. Regarding GMLP, which describes a set of best practices for clinical machine learning, FDA plans to help standardize and create GMLP in collaboration with its Medical Device Cybersecurity program and other communities. Regarding algorithms, FDA is supporting regulatory science research in order to develop methods to increase SaMD robustness.

This action plan would mitigate some issues described in this memo. However, the plan does not explicitly allow the FDA to regulate databases. Instead, its focus is still on regulating the software itself, which is necessary but inherently difficult due to its "black box" nature (i.e., ML software developers can know the input and output that the software should learn from, but by the nature of ML, they cannot fully know the process through which the software makes decisions). In contrast, databases can be managed by introducing regulations for privacy, methods of preventing data silo-ing, and bias mitigation. Further, the quality of the data input directly affects the quality and performance of the software. Regulating at the database level is just as, if not more, important than regulating at the software level. Therefore, we still argue that in order to achieve the most robust regulation of SaMD, Congress should amend the FDCA to allow the FDA to regulate databases.

\section{References}

C. Andel, S. Daviow, M. Hollander and D. Moreno, "The Economics of Health Care Quality and Medical Errors," Journal of Health Care Finance, 2012. https://pubmed.ncbi.nlm.nih.gov/23155743/

T. Araujo, N. Helberger, S. Kruikemeier and C. H. de Vreese, "In AI we trust? Perceptions about automated decision-making by artificial intelligence," AI \& Society, 2020.

https://dare.uva.nl/search?identifier=b73d4d3f8ab9-4b63-b8a8-99fb749ab2c5

"Artificial Intelligence in Healthcare," Academy of Medical Royal Colleges, London, 2019.

https://www.aomrc.org.uk/reportsguidance/artificial-intelligence-in-healthcare/

T. Timan, Z.A. Mann (eds), "Data Protection in the Era of Artifical Intelligence: Trends, existing solutions and recommendations for privacy-preserving technologies", Big Data Value Association, October 2019.

https://www.bdva.eu/sites/default/files/Data\% 20protection\%20in\%20the \%20era\%20of\%20bi g\%20data\%20for\%20artificial\%20intelligence B DVA FINAL.pdf
P. D. Blair, "The evolving role of the US National Academies of Sciences, Engineering, and Medicine in providing science and technology policy advice to the US government," Palgrave Communications, 2016. https://www.nature.com/articles/palcomms201 $\underline{630}$

A. Borkowski, N. Viswanadhan, L. Thoms, R. Guzman, L. Deland, S. Mastorides, "Using Artificial Intelligence for COVID-19 Chest X-ray Diagnosis." Federal Practitioner, pp. 398-404, 2020. https://www.ncbi.nlm.nih.gov/pmc/articles/PM C7535959/

Congress, "S.1108 - Algorithmic Accountability Act of 201," Washington DC, 2019. https://www.congress.gov/bill/116thcongress/senate-bill/1108

J. Downer, "Trust and technology: the social foundations of aviation regulation," The British Journal of Sociology, pp. 83-106, 2010. https://onlinelibrary.wiley.com/doi/abs/10.111 1/j.1468-4446.2009.01303.x

E. Ernst, R. Merola and S. Daniel, "The economics of artificial intelligence: Implications for the future of work," International Labor Office, Geneva, 2018.

https://www.ilo.org/wcmsp5/groups/public/--dgreports/--cabinet/documents/publication/wcms 647306.p $\underline{\mathrm{df}}$ 
R. Gillon, "Medical Ethics: Four Principles Plus Attention to Scope," BMJ, pp. 184-188, 1994. https://www.ncbi.nlm.nih.gov/pmc/articles/PM C2540719/

C. Heneghan. "Early Findings from Fitbit COVID-19 Study Suggest Fitbit Devices Can Identify Signs of Disease at Its Earliest Stages," Fitbit, Inc., Press Release, August 192020 [ Accessed November 12 2020] https://blog.fitbit.com/early-findingscovid-19-study/

International medical Device Regulators Forum (IMDRF) Software as a Medical Device (SaMD) Working Group. "Software as a Medical Device (SaMD): Key Definitions", IMDRF, 2013. http://academy.gmpcompliance.org/guidemgr/files/IMDRF-TECH131209-SAMD-KEY-DEFINITIONS.PDF

J. A. Kroll, J. Huey, S. Barocas, E. W. Felten, J. R. Reidenberg, D. G. Robinson and H. Yu, "Accountable Algorithms," University of Pennsylvania Law Review, vol. 165, 2017. https://scholarship.law.upenn.edu/penn law rev iew/vol165/iss $3 / 3 /$

J. Laguarta, "COVID-19 Artificial Intelligence Diagnosis using only Cough Recordings," IEEE, 2020. https://ieeexplore.ieee.org/document/9208795

Library of Congress, "Regulation of Artificial Intelligence: The Americas and the Caribbean," 1 July 2019. [Online]. Available: https://www.loc.gov/law/help/artificialintelligence/americas.php [Accessed 31 March 2020].

J. C. Moskop, C. A. Marco, G. L. Markin, J. M. Geiderman and A. R. Derse, "From Hippocrates to HIPAA: Privacy and Confidentiality in Emergency Medicine - Part I: Conceptual, Moral, and Legal Foundations," Annals of Emergency Medicine, pp. 53-59, 2005. https://pubmed.ncbi.nlm.nih.gov/15635311/

National Academies of Science, Engineering, Medicine, "Public Health Consequences of E-Cigarettes," 2018.

https://pubmed.ncbi.nlm.nih.gov/29894118/

National Academies of Sciences, Engineering, and Medicine, "Standing Committee on Emerging Infectious Diseases and 21st Century Health Threats," 2020. [Online]. Available: https://www.nationalacademies.org/ourwork/standing-committee-on-emerginginfectious-diseases-and-21st-century-healththreats [Accessed 31 March 2020].

"National Defense Authorization Act of 2019, Section 238," Washington DC, 2018. https://www.congress.gov/115/bills/hr5515/BI LLS-115hr5515enr.pdf
W. N. I. Price, "Artificial Intelligence in Health Care: Applications and Legal Issues," The SciTech Lawyer, pp. 10-13, 2017. https://repository.law.umich.edu/cgi/viewconte nt.cgi?article $=2932 \&$ context $=$ articles

Results from search on Congress.gov using the phrase "artificial intelligence", 2020.

Y. R. Shrestha and S. M. v. K. G. Ben-Menahem, "Organizational Decision-Making Structures in the Age of Artificial Intelligence," California Management Review, pp. 66-83, 2019. https://journals.sagepub.com/doi/abs/10.1177/ 0008125619862257 ? journalCode $=\mathrm{cmra}$

US Food and Drug Administration, "A History of Medical Device Regulation and Oversight in the United States," 24 June 2019. [Online]. Available: https://www.fda.gov/medical-devices/overviewdevice-regulation/history-medical-deviceregulation-oversight-united-states [Accessed 31 March 2020].

US Food and Drug Administration (FDA), "Proposed Regulatory Framework for Modifications to Artificial Intelligence/Machine Learning (AI/ML)Based Software as a Medical Device (SaMD)," 2019.

https://www.fda.gov/files/medical\%20devices/ published/US-FDA-Artificial-Intelligence-andMachine-Learning-Discussion-Paper.pdf

US Food and Drug Administration (FDA), "Selected Amendments to the FD\&C Act," 29 March 2018. [Online]. Available: https://www.fda.gov/regulatoryinformation/laws-enforced-fda/selectedamendments-fdc-act [Accessed 31 March 2020].

US Food and Drug Administration (FDA), "Title 21 Code of Federal Regulations Parts 800-1299”, 11 November 2020. [Online]. Available: https://www.accessdata.fda.gov/scripts/cdrh/cf docs/cfcfr/CFRSearch.cfm?CFRPartFrom $=800 \& \mathrm{C}$ FRPartTo=1299 [Accessed 12 January 2021].

US Food and Drug Administration (FDA), "Artificial Intelligence/Machine Learning (AI/ML)-Based Software as a Medical Device (SaMD) Action Plan", January 2021. [Online]. Available: https://www.fda.gov/medical-devices/softwaremedical-device-samd/artificial-intelligence-andmachine-learning-software-medical-device. [Accessed 12 January 2021]. 
Nicole Bonan is a 3rd year PhD student studying cancer biology at the George Washington University. Her dissertation research focuses on genetically modifying NK cells and administering tumor stroma-degrading enzymes to increase immune cell penetration and killing of solid tumors. She is interested in adoptive cell therapy, nanomedicine, and science policy. She received her MS in biology and BS in biochemistry from American University.

Jaclyn Brennan is a postdoctoral researcher in the Biomedical Engineering Department at GWU. She received her PhD from GWU in 2020, with a research focus in cardiac electrophysiology and arrhythmias of the cardiac conduction system. She hopes to eventually apply her technical knowledge and problem-solving skills toward a career in science policy.

Anthony Hennig is a 5th year $\mathrm{PhD}$ candidate studying engineering complexity as part of the systems engineering program in the Department of Engineering Management and Systems Engineering. They graduated with a Master of Science in Science, Technology, and Public Policy from the Rochester Institute of Technology in 2016 and they are interested in aerospace technology policy as well as program and project management with respect to public policy.

Mark Alexander Kaltenborn is a 5 th year PhD student in the Department of Physics at the George Washington University (GWU) and a graduate student researcher in the Computational Physics and Methods Group at Los Alamos National Laboratory. He conducts numerical studies of white dwarf and neutron star mergers with particle-based hydrodynamics codes and develops the Advanced Simulation and Computing Program Ristra Project's FleCSPH code. Alexander has completed the International Science and Technology Policy graduate certificate program at GWU and continues to pursue his interest in science policy.

\section{Acknowledgements}

The authors would like to acknowledge the organizers of the National Science Policy Network's Science Policy Memo workshop for providing an environment in which the authors could receive feedback on and refine this memorandum.

\section{Disclaimer}

The authors declare no conflicts of interest. 